\title{
Influencia familiar, factor decisivo en la conducta de los estudiantes en la enseñanza básica Influencia familiar en la enseñanza básica
}

Uber Constantino Guerrero Mantuano. Mg. Sc Instituto Tecnológico Superior Portoviejo. Ecuador

Contacto: hcguerrero@hotmail.com

Receptado: $12 / 07 / 2014 \quad$ Aceptado: 04/010/2014

\section{Resumen}

La familia tiene el impacto directo y duradero en el aprendizaje y desarrollo educativo de sus hijos. Este aspecto es, actualmente, una problemática muy investigada por muchos pedagogos en Ecuador. Hoy en día, los problemas educativos más cruciales presentados en los centros de estudios en Ecuador estriban en el papel que juega la familia en la formación del estudiante. El objetivo de la investigación fue analizar los procesos en que se ven implicados los problemas conductuales de los estudiantes del centro Educativo Felipe Intriago Bravo. Para el desarrollo del trabajo se utilizó bibliografía de muchos estudiosos del tema, que sirvieron de apoyo a los métodos empleados, donde se destacan el deductivo, el cualitativo y cuantitativo. Se concluyó que el papel que juega la familia en la erradicación problemas conductuales de los estudiantes ayuda a la formación de estos, que al mismo tiempo son parte de la familia. El rol de los padres contribuye al mejoramiento de los resultados académicos, así como al progreso actitudinal de los estudiantes hacia las tareas escolares y de hecho a su formación integral.

Palabras claves: familia, comportamiento, problemas de conductas, formación, aprendizaje, desarrollo educativo

\section{Family influence, a decisive factor in the behavior of students in basic education} \begin{abstract}
The family has a direct and lasting impact on learning and educational development of their children. This aspect is a present-day problem investigated by pedagogues in Ecuador. Today, the most crucial educational problems presented in the school in Ecuador lie in the role of the family in student education. The aim of the research was to analyze the processes that are involved in the behavioral problems of students School "Felipe Intriago Bravo". For the development it was used the literature of many investigators, who served in support of the
\end{abstract}


Rev. SINAPSIS, Vol. 5, No 2, Diciembre 2014

ISSN $1390-7832$

methods used, highlighting the deductive, qualitative and quantitative ones. It was concluded that the role of the family in the eradication of the behavioral problems of students helps the formation of theme, who are at the same time, part of the family. The role of parents contributes to improving academic outcomes, as well as attitudinal student progress toward school tasks and their integral formation.

Keywords: family, behavior, behavior problems, training, learning, educational development

\section{Introducción}

"Desde el momento del nacimiento la familia tiene un papel esencial en el desarrollo de las personas...es la familia la que garantiza, además de la supervivencia física, los aprendizajes básicos para el desenvolvimiento autónomo dentro de la sociedad”. (Maestre, 2009, p. 8)

La influencia de la familia en el comportamiento y desarrollo de la personalidad constituye un hecho ampliamente reconocido y estudiado por las diversas disciplinas que analizan y valoran la conducta humana. El hecho de que esta influencia pueda degenerar en modos de comportamiento que son expresión de un desarrollo inadecuado de los individuos y lleva consigo una disfunción evidente de sus roles específicos es, sin duda un problema que han de afrontar cotidianamente los Trabajadores Sociales y profesionales en la orientación y educación

En concordancia con lo expuesto, estudios a nivel mundial han mantenido una atención constante al estudio de la incidencia que tienen los factores afectivos familiares, en la vida humana. Según Soto (2013). "La Familia como núcleo básico de la sociedad es quien se encarga de impartir valores a sus miembros. Aquí se destaca en particular el hecho de que los trastornos emocionales que provoca en el niño un ambiente familiar poco acogedor o deficientemente cohesionado se agudizan, sin duda, cuando en él se viven situaciones de conflicto o tensión que degeneran en la agresión o la violencia y el niño queda expuesto a los malos tratos que se derivan generalmente de ellas.

El Centro Educativo "Felipe Intriago Bravo" del cantón Portoviejo que es la institución educativa donde se encuentra la población objeto de estudio, no está excepta de esta problemática, especialmente en los estudiantes de quinto a noveno grado, donde un porcentaje significativo de ellos tienen problemas conductuales que ameritan un estudio objetivo que permita identificar las causas, especialmente aquellas que están estrictamente relacionadas con el rol de la familia en el proceso enseñanza aprendizaje y el rol del docente 
como orientador y facilitador de este proceso, son innumerables los factores a los que se atribuye esta problemática, entre los cuales de acuerdo a la investigación previa, tienen mayor incidencia los hogares disfuncionales y el escaso interés de los docentes por la aplicación de procesos apropiados para la resolución de conflictos disciplinarios; factores que están íntimamente ligados con el nivel de comunicación hogar-escuela.

El presente trabajo de investigación tiene como objeto de estudio "La familia y su incidencia en el comportamiento de los estudiantes de educación media y superior del centro educativo "Felipe Intriago Bravo".

De forma general se presenta los resultados que se obtuvieron en la investigación, considerando como hecho más relevante que, tanto los docentes como el Director de la institución educativa mantienen un buen nivel de comunicación con la mayoría de los padres y/o madres de familia, esto de alguna manera permite que los problemas de conducta que presentan los estudiantes siempre se los trate de resolver de la forma más adecuada y pertinente tomando como referente de guía el Código de Connivencia, documento legal que no especifica el proceso a seguir para abordar los casos de indisciplina o de conducta que ameriten tomar otras medidas o correctivos establecidos de acuerdo a la LOEI, por lo que se concluye que es necesario implementar en la institución un Manual de Procedimiento específico para tratar estos casos.

El objetivo de la investigación fue analizar los procesos en que se ven implicados los problemas conductuales de los estudiantes del centro Educativo Felipe Intriago Bravo. Este análisis se convierte en una herramienta de gran utilidad para que directivos y docentes cuenten con un referente teórico y práctico para atender de forma adecuada los problemas de conducta de los estudiantes y sirve además, como base a futuras investigaciones.

\section{Materiales y métodos.}

Para el comienzo de la investigación se tomó como referencia documental la bibliografía de algunos estudiosos del tema, donde se utilizó, además, información de textos, revistas, artículos publicados en la internet, investigaciones, documentos legales entre otros que guardan relación directa con la problemática de estudio. 
Rev. SINAPSIS, Vol. 5, No 2, Diciembre 2014

Para obtención de los criterios iniciales prácticos y el posterior tratamiento al problema investigativo, se efectuaron encuestas y entrevistas con objetivos marcados, que permitieron una visión general de los procesos y procedimientos utilizados para dilucidar el papel de la familia en la educación de sus hijos y la estrecha relación que estos deben guardar con la escuela.

En el trabajo se utilizaron diferentes métodos como el deductivo que sirvió para formar una base sólida en el planteamiento lógico de la hipótesis. Se utilizó también el método cualitativo que fue de gran importancia para realizar una interpretación más amplia y analítica de los resultados, pues se considera cada una de los factores que están relacionados con el tema y el cuantitativo a través del cual y con la ayuda de la estadística se logró obtener una apreciación cuantitativa y medible de los resultados y de la verificación de la hipótesis.

\section{Desarrollo}

Con el objetivo de establecer la influencia de los hogares disfuncionales en la práctica de los principios de autoridad en el hogar se encuestó a un grupo de padres de la Centro Educativo Felipe Intriago Bravo.

\section{CUADRO $\mathbf{N}^{\circ} 1$}

1. ¿Quiénes viven en su hogar?

\begin{tabular}{c|lcc} 
ORDEN & ALTERNATIVAS & F & \% \\
\hline a. & Papá-Mamá-Hermanos & $\mathbf{3 0}$ & $\mathbf{4 1 , 6 6}$ \\
\hline b. & Papá y hermanos & $\mathbf{0}$ & $\mathbf{0}$ \\
c. & Mamá y hermanos & $\mathbf{3 9}$ & $\mathbf{5 4 , 1 6}$ \\
d. & Otros parientes & $\mathbf{3}$ & $\mathbf{4 , 1 6}$ \\
e. & Sólo hermanos & $\mathbf{0}$ & $\mathbf{0}$ \\
f. & Vive solo & $\mathbf{0}$ & $\mathbf{0}$ \\
& TOTAL & $\mathbf{7 2}$ & $\mathbf{9 9 . 9 9}$ \\
\hline
\end{tabular}

\section{GRÁFICO No 1}

\section{ESTRUCTURA DEL HOGAR}

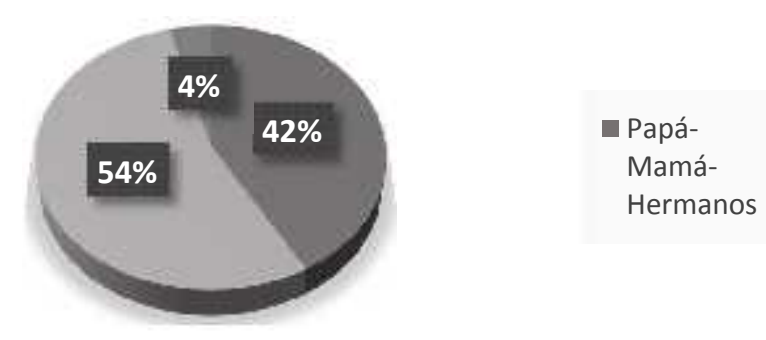


Rev. SINAPSIS, Vol. 5, N² 2, Diciembre 2014

ISSN $1390-7832$

Se indagó a los padres y/o madres de familia sobre la estructura familiar, los resultados que se obtuvieron se comportaron de la siguiente forma: 30 de los padres encuestados que representan el 41, 66 de los padres y/o madres encuestados(as), respondieron Papá-MamáHermanos, 39 de ellos que corresponde al 54,16\% señalaron la alternativa: mamá y hermanos y 3 que son el $4,16 \%$ indicaron que viven con otros parientes.

Los resultados demostraron que la mayoría de las familias tiene como miembros de la familia a mamá y hermanos, así mismo que también existe un número significativo de familias cuyos miembros son papá, mamá y hermanos.

Para Piaget (1971, p. 64) La familia cumple su rol instaurador de posibilidades, deja la primera huella de lo que será el desarrollo intersubjetivo en la sociedad. Los niños aprenden todo fácilmente, incluso cómo ser aquello de lo que él se espera y responde de esta manera a los deseos no solo explícitos sino a los implícitos del adulto.

Uno de los elementos esenciales en el proceso formativo de un niño, es precisamente el apoyo familiar, apoyo que está sujeto a la influencia directa de aspectos determinantes como su estructura, generalmente no reciben la misma atención ni tienen las mismas posibilidades los niños cuya estructura familiar esta resquebrajada por la falta de unos de sus miembros; el rol instaurador de la familia del que habla Piaget de alguna manera se debilita generando como consecuencia un déficit de atención que desencadena otros problemas como mal comportamiento e indisciplina, actitud que refleja a diario en el aula de clase.

\section{CUADRO $\mathrm{N}^{\circ} 2$}

¿Quiénes ejerce la primera autoridad en su hogar?

\begin{tabular}{cccc} 
ORDEN & ALTERNATIVAS & F & \% \\
\hline a. & Papá & $\mathbf{2 8}$ & $\mathbf{3 8 , 8 8}$ \\
\hline b. & Mamá & $\mathbf{4 2}$ & $\mathbf{5 8 , 3 3}$ \\
\hline c. & Papá - Mamá & & \\
\hline d. & Otros parientes & $\mathbf{2}$ & $\mathbf{2 , 7 7}$ \\
e. & Todos & & \\
f. & Ninguno & & \\
& TOTAL & $\mathbf{7 2}$ & $\mathbf{9 9 . 9 9}$ \\
\hline
\end{tabular}

\section{GRÁFICO No 2}




\section{PRIMERA AUTORIDAD DEL HOGAR}

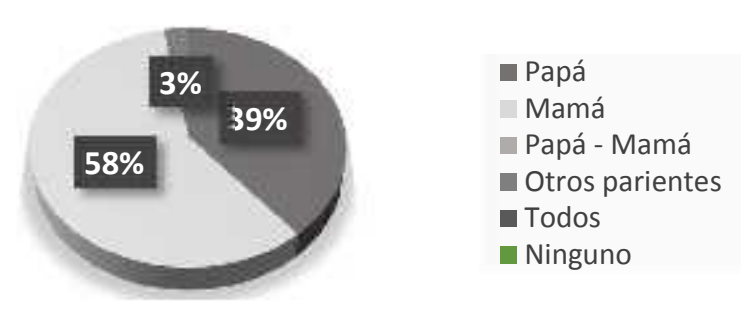

Esta pregunta se la formuló a los padres con el objetivo de tener una apreciación de cómo ellos consideran el ejercicio de autoridad en el hogar, se platearon seis alternativas y se obtuvieron los siguientes resultados: 28 padres que corresponden al 39\% señalaron que el ejercicio de primera autoridad lo tiene el papá; 42 de ellos que representan el 58\% señalaron que lo consideran que esta función la ejerce mamá y dos de los padres y/o madres que son el $3 \%$ de los padres encuestados indicaron que el rol de primera autoridad lo ejerce otro familiar.

Las respuestas obtenidas permiten establecer que en la mayoría de los hogares, el rol de la primera autoridad lo ejerce la madre de familia, hecho que responde de alguna manera a la estructura que tienen la mayoría de las familias involucradas en el estudio.

Para Vygotsky (1997, p. 38), "el comportamiento es una forma de apropiación de la herencia cultural disponible, no sólo es un proceso individual de asimilación. La interacción social es el origen y el motor del aprendizaje"

Uno de los hechos que actualmente caracteriza a un porcentaje significativo de familias manabitas, es precisamente su disfuncional estructura, es decir, que la mayoría de estas no está integrada por todos sus miembros, especialmente en el caso de los padres, es así que la madre ha adoptado un comportamiento bastante autónomo e independiente, que la hace figurar como la persona que ejerce el principio de autoridad en el hogar y que de acuerdo a Vygotsky le permite desarrollar otras destrezas y conocimiento que pasan a ser parte de su cultura y por ende la de los niños.

\section{CUADRO No 3}

3. ¿Considera que la ausencia del padre o la madre en el hogar puede influir en el comportamiento de los hijos 
Rev. SINAPSIS, Vol. 5, No 2, Diciembre 2014

ISSN $1390-7832$

\begin{tabular}{c|lcc} 
ORDEN & ALTERNATIVAS & F & $\%$ \\
\hline a. & Siempre & $\mathbf{6 4}$ & $\mathbf{8 8 , 8 8}$ \\
b. & A veces & 11 & $\mathbf{1 5 , 2 7}$ \\
\hline c. & Rara vez & & \\
\hline d. & Nunca & & \\
& TOTAL & 72 & \\
\hline
\end{tabular}

\section{GRÁFICO No 3 \\ INFLUENCIA DE AUSENCIA DE LOS PADRES \\ EN EL COMPORTAMIENTO DE LOS HIJOS}

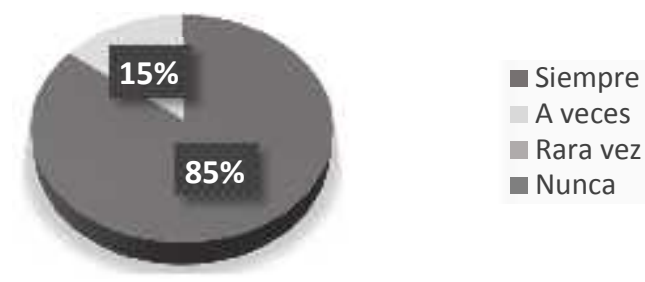

Esta pregunta se la realizó con el objetivo de indagar sobre la apreciación que tienes los padres en cuanto a la influencia que tienen el comportamiento de los hijos la ausencia de uno de ellos en el hogar, los resultados obtenidos fueron: en la primera opción que es siempre, se obtuvo el $89 \%$ que representan a 64 padres de familia, $15 \%$ que equivale a 11 padres y/o madres de familia encuestados contestaron que poco las opciones $\mathrm{c} y$ de no fueron seleccionadas.

De acuerdo a los resultados se puede establecer que la mayoría de los padres consideran que la ausencia de cualquiera de los padres influye directamente en el comportamiento de los hijos

Vygotsky (1978 p. 77), destacó "el valor de la cultura y el contexto social, que veía crecer el niño a la hora de hacerles de guía y ayudarles en el proceso de aprendizaje. El niño tiene un papel activo en el proceso de aprendizaje pero no actúa solo".

Lo que expresa Vygotsky, ratifica la apreciación que tienen los padres sobre la influencia que tiene la ausencia de uno de ellos en el comportamiento de los hijos; de acuerdo a lo que expresa el autor y a la experiencia que se tiene como docente y padre de familia, es acertado 
que el entorno familiar juega un papel determinante en los niños, pues se convierten en guía, en patrones a seguir tal es así que la falta de uno de ellos en el seno familiar de alguna forma afecta su formación, específicamente en cuanto a comportamiento, el mismo que evidencia en qué medida y en qué aspectos le ha afectado la falta de papá, mamá o de ambos.

\section{CUADRO No 4}

¿Quiénes viven en su hogar?

\begin{tabular}{cllc} 
ORDEN & ALTERNATIVAS & F & \% \\
\hline a. & Papá-Mamá- & $\mathbf{4 2}$ & $\mathbf{4 2}$ \\
& Hermanos & & \\
b. & Papá y hermanos & & \\
c. & Mamá y hermanos & $\mathbf{4 9}$ & $\mathbf{4 9}$ \\
\hline d. & Otros parientes & $\mathbf{9}$ & $\mathbf{9}$ \\
e. & Sólo hermanos & & \\
f. & Vive solo & & \\
\hline
\end{tabular}

Se plantea esta interrogante a los estudiantes para verificar aspectos familiares como la estructura, obteniéndose como resultados que 42 estudiantes que refleja el $42 \%$ indicaron que viven con papá, mamá y hermanos; 49 que representan el $49 \%$ señalaron que viven con la mamá y sus hermanos y nueve estudiantes que constituyen el $9 \%$ respondieron que con otros familiares

Las respuestas obtenidas ratifican los resultados obtenidos de los padres de familia, es decir la mayoría de los estudiantes viven solo con la mamá y los hermanos. De igual forma no se puede obviar que existen un porcentaje significativo de estudiantes que si viven en un hogar estructurado por papá, mamá y hermanos.

Piaget (1952, p.68) "consideraba que los niños dan sentido a las cosas principalmente a través de sus acciones en su entorno", Vygotsky (1978 :76) destacó el valor de la cultura y el contexto social, que veía crecer el niño a la hora de hacerles de guía y ayudarles en el proceso de aprendizaje.

Lo expuesto por Piaget y Vygotsky ratifica la importancia que tiene la estructura familiar como un elemento clave y determinante para una formación adecuada y normal del niño o la niña, el entorno familiar se convierte en el agente social que le proporciona y trasmite experiencias, valores y conocimientos que formarán parte de su identidad y personalidad. 


\section{CUADRO $\mathrm{N}^{\circ} 5$}

\section{¿Quién ejerce la primera autoridad en el hogar?}

\begin{tabular}{c|lcc} 
ORDEN & ALTERNATIVAS & F & $\mathbf{\%}$ \\
\hline a. & Papá & $\mathbf{4 0}$ & $\mathbf{4 0}$ \\
\hline b. & Mamá & $\mathbf{4 9}$ & $\mathbf{4 9}$ \\
\hline c. & Papá - Mamá & & \\
\hline d. & Otros parientes & $\mathbf{1 1}$ & $\mathbf{1 1}$ \\
e. & Todos & & \\
f. & Ninguno & & \\
\hline
\end{tabular}

Se preguntó a los estudiantes involucrados en el estudio sobre su consideración en cuanto a quien ejerce la primera autoridad en casa, las respuesta fueron: el $40 \%$ de los encuestados que representan a 40 estudiantes respondieron que el papá 49\% de los encuestados correspondientes a 49 estudiantes indicaron que consideran que la primera autoridad la ejerce la mamá, y el $11 \%$ de la población estudiantil encuestada que equivale a 11 estudiantes indicaron que otros familiares.

Los resultados obtenidos corroboran la información proporcionada por los padres de familia, se puede establecer entonces que en la mayoría de los hogares es la mamá quien ejerce la primera autoridad.

Vygotsky (1991, p.17), también destacó "la importancia del entorno familiar, especialmente de la counicación en el desarrollo cognitivo, demostrando que si los niños disponen de estos elementos, son capaces de construir conceptos mucho más rápidamente. Creía que el pensamiento y el lenguaje convergían en conceptos útiles que ayudan al pensamiento. Observó que el lenguaje era la principal vía de transmisión de la cultura y el vehículo principal del pensamiento y la autorregulación voluntaria »

Lo expuesto por Vygostky se puede corroborar en la respuesta emitida por los estudiantes, al tener su propio concepto y pensamiento sobre el ejercicio de autoridad, es así como ellos a través de la interacción social en el seno mismos de la familia han logrado interpretar y fijarse un concepto que responde a su realidad pues la mayoía de los hogares son difuncionales donde obviamente quien lleva la mayor responsabilidad y conduce el hogar es la madre.

\section{CUADRO $\mathrm{N}^{\circ} 6$}

¿Considera que la ausencia del padre o la madre en el hogar puede influir en el comportamiento de los hijos 


\begin{tabular}{c|ccc|} 
ORDEN & ALTERNATIVAS & F & $\%$ \\
\hline a. & Siempre & 75 & 75 \\
\hline b. & A veces & 17 & 17 \\
\hline c. & Rara vez & 8 & 8 \\
\hline d. & Nunca & & \\
\hline & TOTAL & 100 & 100 \\
\hline
\end{tabular}

Esta interrogante se planteó a los estudiantes para conocer la apreciación que tienen sobre la influencia que ejerce sobre su comportamiento la ausencia de uno de los padres, las respuestas encontradas fueron las siguientes: que 75 estudiantes que representan el $75 \%$ contestaron que siempre, 17 estudiantes que equivale el $17 \%$ respondieron que a veces, y ocho estudiantes que representa un $8 \%$ contestaron que rara vez.

De acuerdo a los resultados obtenidos se puede establecer que la mayoría de los estudiantes consideran que la ausencia de uno de los padres si afecta directamente en su compartimiento corroborando con esto la respuesta emitida por los padres de familia.

Vigotsky (1979, p.97) señala que para descubrir las relaciones reales del proceso evolutivo con las aptitudes de aprendizaje se tiene que delimitar como mínimo dos niveles evolutivos. El primero de ellos lo denomino nivel evolutivo real, es decir, el nivel de desarrollo de las funciones mentales de un niño, establecido como resultado de ciertos ciclos evolutivos, estas son aquellas actividades que el niño puede realizar por si solo sin ninguna ayuda. El segundo nivel Vigotsky lo denomino zona de desarrollo próximo, es decir, la distancia entre el nivel real de desarrollo y el nivel de desarrollo potencia, determinado a través de la resolución de un problema bajo la guía de un adulto o en colaboración con otro compañero más capaz, son actividades que todavía no han madurado, pero que se hallan en proceso de maduración.

Al relacionar la apreciación que tienen los estudiantes en cuanto a la influencia de la ausencia de uno de sus padres con el segundo nivel de aprendizaje conocido como Zona de Desarrollo próximo que expone Vygotsky en la que los niños requiere del acompañamiento de un adulto o un compañero para lograr potenciar su aprendizaje. En el contexto educativo esta podría ser una de las influencias más significativas a la que seguramente se refieren los estudiantes porque esta falta de atención deriva a su vez otros problemas como la falta de hábitos de estudio, indisciplina, mal comportamiento y actitudes negativas hacia los compañeros y en casos extremos hasta los docentes, quienes en algunos casos no saben qué medidas correctivas 
Rev. SINAPSIS, Vol. 5, No 2, Diciembre 2014

tomar, específicamente en los casos de estudiantes reincidentes con los que ya se han agotado diferentes medidas para abordar y tratar este problema, que a opinión de ellos requiere de suma urgencia un tratamiento diferente, que haga reflexionar al estudiante y a los padres y/o madre de familia de quienes se requiere un mayor grado de compromiso en relación a la atención de este problema.

\section{CUADRO No 7}

1. ¿Quiénes viven en los hogares de sus estudiantes?

\begin{tabular}{clcc} 
ORDEN & ALTERNATIVAS & F & $\%$ \\
\hline a. & Papá-Mamá- & $\mathbf{3}$ & $\mathbf{2 5}$ \\
\hline b. & Hermanos & & \\
c. & Mamá y hermanos y hermanos & $\mathbf{8}$ & $\mathbf{6 6 , 6 6}$ \\
\hline d. & Otros parientes & $\mathbf{1}$ & $\mathbf{8 , 3 3}$ \\
e. & Sólo hermanos & & \\
f. & Vive solo & & \\
\hline
\end{tabular}

Respecto a esta pregunta los docentes respondieron: tres de ellos que son el $25 \%$ respondieron que de acuerdo a la ficha de registro en los hogares de los estudiantes viven, papá-mamá-hermanos; ocho de os docentes a quienes se les asigna el 67\% indicaron que de acuerdo a su experiencia y registro sus estudiantes viven con la mamá y de ellos que representa el $8 \%$ de los docentes encuestados señaló que en su registro aparecen algunos estudiantes que viven con otros familiares.

De acuerdo a las respuestas emitidas por los docentes se corrobora una vez más que la mayoría de los estudiantes viven con la mamá y hermanos.

Piaget (1959), establece etapas para el desarrollo cognoscitivo: sensoriomotor, preoperacional, operaciones concretas y operaciones formales, por su parte la teoría de Vigotsky establece la zona de desarrollo próximo. Para Piaget los mecanismos de aprendizaje son : la asimilación, la acomodación y el equilibrio, mientras que para Vigotsky, el aprendizaje y el desarrollo son una actividad social y colaborativa que no puede ser "enseñada" a nadie. Depende del estudiante construir su propia comprensión en su propia mente. 
Rev. SINAPSIS, Vol. 5, No 2, Diciembre 2014

ISSN $1390-7832$

Con las diferentes encuestas que realizaron se obtuvieron suficientes criterios para poseer un estado analítico de planteamiento del problema, criterios que sirvieron de base para la propuesta un manual de procesos para atender los problemas conductuales de los estudiantes.

Mediante el análisis observacional se corroboró que la los aspectos concernientes a la relación padre-escuela. La cooperación entre padres y escuela puede resultar difícil, Fish (1990) clasificó las dificultades de estas relaciones en cuatro grandes bloques: filosóficas, actitudinales, problemas de logística y habilidades deficientes. Los padres y los profesores expresaron una serie de dificultades respecto a la participación de los padres en las escuelas García-Bacete, (2003, p.45).

Se pudo probar que la relación entre los dos elementos era débil. Se constató que la mayoría de los padres desconocían todo lo relacionado con el entorno escolar, que tenían dificultades en la compresión de la legislación y de la organización del sistema educativo, que algunos padres pretendían dirigir al profesorado, que podía existir una interferencia de intereses particulares en los padres...Por otro lado, los profesores dieron argumentos del tipo: algunos padres intentan imponer sus ideas, interfieren en nuestra labor, sólo generan problemas, los padres no saben cómo funcionan las escuelas, consideran que determinadas metodologías son tonterías...

Parte del problema, como señalan Terrón, Alfonso y Díez (2003), reside en que los canales de comunicación establecidos legalmente entre la familia y la escuela no son efectivos realmente y que la falta de un clima de confianza entre ambos sectores mina toda $\mathrm{p}$ cación positiva y efectiva.

Se confirmó que unaa de las grandes dificultades de los padres y maestros es saber cómo manejar la conducta inadecuada o "conducta-problema" de sus niños. A menudo los escuchamos decir frases como: "no me hace caso", "no quiere hacer lo que se le dice", "se molesta cuando le impedimos algo", "hace berrinches", "no acepta límites", "ya no sabemos qué hacer frente a su desobediencia y su rebeldía" etc., de allí que es importante que los maestras y/o padres adquieran recursos para enfrentar estas situaciones, y tomen conciencia que la mayor parte de estos comportamientos son aprendidos. Está definida como la actividad 
Rev. SINAPSIS, Vol. 5, No 2, Diciembre 2014

ISSN $1390-7832$

global de un organismo que hace posible la adaptación a su medio específico y que le proporciona control e independencia frente ha dicho medio.

En ese sentido, es importante recordar que los niños no heredan buenos ni malos hábitos, su conducta es el resultado del aprendizaje en el ambiente familiar, escolar y social; sin embargo, también hay que tener en cuenta que las conductas pueden ser "desaprendidas" o cambiadas.

Ignorar Activamente Implica el retiro total e inmediato de los diferentes reforzadores que pueden estar manteniendo la conducta. Se aplica en todo tipo de conductas-problema, pero sin manifestaciones agresivas. Tiempo de Reflexión consiste en retirar al niño, por unos momentos de la situación o contexto en el que se lleva a cabo la conducta-problema, con la finalidad de que no se refuerce la conducta.

El lugar de ubicación del niño podrá ser un sector del aula u otro ambiente. Reforzamiento de Conductas Adecuadas, en el repertorio conductual de un niño podemos encontrar una variedad de conductas inadecuadas, pero a la vez existirán conductas positivas, incompatibles con ellas, física y temporalmente.

El adulto debe reforzar positivamente sólo las conductas adecuadas y no las conductas socialmente indeseables. Sobre corrección es una técnica que se usa cuando la conductaproblema ha causado un daño observable en el medio ambiente, y consiste en corregir los efectos producidos por la misma, además de ejecutar una actividad extra que implique la realización de un comportamiento apropiado.

Puede emplearse en casos de conductas agresivas y auto estimulatorias. Sanciones Lógicas consiste en administrar una consecuencia desagradable inmediatamente después de ocurrida la conducta problema. Se utiliza para reducir conductas inadecuadas. Y Pérdida de Privilegios, es la retirada de un estímulo reforzante ya ganado, que obra en el poder del niño en el momento de emitir la respuesta indeseable.

\section{Discusión}

La influencia de los hogares disfuncionales en la práctica de los principios de autoridad en el hogar, en la mayoría de los casos está relacionada directamente con la estructura familiar, pues de acuerdo a los resultados obtenidos en la investigación se pudo conocer que la mayoría de los hogares son disfuncionales, formados por mamá e hijos, por lo tanto, es la madre quien ejerce los principios de autoridad; mientras que en los hogares cuya estructura es 
Rev. SINAPSIS, Vol. 5, No 2, Diciembre 2014

ISSN $1390-7832$

completa: papá, mamá e hijos el principio de autoridad lo ejerce el padre. Este último hallazgo encontrado conduce a pensar que en la mayoría de los hogares funcionales o completos aún se mantiene una cultura machista en cuanto al ejercicio del principio de autoridad, pues es solo el papá a quien los hijos le asignan este derecho, avizorando desde ya una herencia cultural machista para el niño/a.

Las relaciones interpersonales y el trabajo en equipo que los estudiantes, mantienen en el aula, en lo que se refiere de forma general a las relaciones interpersonales entre compañeros, se las puede determinar como buenas, no así cuando específicamente requieren trabajar en equipo, en ese momento de acuerdo a criterio del docente no logran trabajar de forma ordenada, ni de forma cooperativa, especialmente aquellos estudiantes que provienen de hogares disfuncionales.

\section{Conclusión}

Este trabajo investigativo centrado en el análisis concluyó en que las relaciones escuela familia son de gran importancia para la formación integral de los estudiantes por lo que se debe establecer una relación de cooperación entre ambas instituciones, ya que las dos buscan la formación y desarrollo del mismo objetivo los estudiantes. Es tendrán que entrar en interacción para unificar y complementar criterios, aunar medios y esfuerzos, de modo que trabaje con los aspectos conductuales para que se pueda percibir una continuidad entre la educación que reciben en el centro y la que reciben en la familia.

\section{Bibliografía}

1. Kerr C y Bowen M. (1988), "Evaluación familia de Michael E

2. Maestre, B. (2009). La familia y la escuela. Los pilares de la educación. Innovavion y experiencias educativas, recuperado el 22 de marzo de 2014 en http://www.csicsif.es/andalucia/modules/mod_ense/revista/pdf/Numero_14/ANA\%20 BELEN_MAESTRE_1.pdf

3. Soto, L. (2013). La familia como núcleo básico de la sociedad. Familia y Educación. Recuperado el 29 de julio de 2014. En http://www.upch.edu.pe/tropicales/ AIDSITS/comunidad/comunidad.pdf

4. Piaget, Jean. (1960),"La nueva educación moral. Editorial Losada S.A., Buenos Aires" 
Rev. SINAPSIS, Vol. 5, No 2, Diciembre 2014

5. Kerr C y Bowen M. (1988), "Evaluación familia de Michael E 